\title{
Vasopressin in Persistent Pulmonary Hypertension of Newborn
}

\author{
Deepak Chawla $^{1}$ (iD
}

Received: 27 February 2021 / Accepted: 8 March 2021 / Published online: 26 March 2021

(C) Dr. K C Chaudhuri Foundation 2021

Persistent pulmonary hypertension of newborn (PPHN) is failure of reduction of pulmonary vascular pressure at birth resulting in decreased pulmonary blood flow and hypoxic respiratory failure. Although PPHN can result from underdevelopment (e.g., in congenital diaphragmatic hernia) or maldevelopment (e.g., excessive vascular muscularization) of the pulmonary vasculature, more commonly it is caused by maladaptation [1]. In the latter, pulmonary vasculature is normal but remains abnormally constricted after birth in response to perinatal conditions like meconium aspiration syndrome or congenital pneumonia, which interfere with normal transition from fetal to neonatal circulation. Diagnosis of PPHN requires high degree of clinical suspicion and access to measurement of oxygenation by pulse oximetry or arterial blood gas. In the absence of right-to-left shunting at the level of ductus arteriosus, the absence of differential oxygenation between upper and lower body cannot rule out PPHN, and the confirmation of diagnosis needs echocardiography. In the absence of a diligent approach and availability of point-of-care echocardiography, PPHN can be both under- and overdiagnosed. Due to higher incidence of risk factors like intrauterine growth restriction, birth asphyxia, meconium aspiration syndrome, and congenital pneumonia, secondary PPHN due to maladaptation is commoner in resourcelimited settings $[2,3]$. However, use of inhaled nitric oxide (iNO), the selective pulmonary vasodilator and the only approved specific therapy for PPHN, is limited to select few centers due to need of specialized delivery equipment and trained healthcare personnel and high initial and recurring cost. As a result, intravenous or oral sildenafil and, to a lesser extent, milrinone infusion have been used as main therapeutic agents for management of PPHN in resource-limited settings. In addition, even when iNO is administered, clinical response

Deepak Chawla

drdeepakchawla@gmail.com

1 Department of Neonatology, Government Medical College Hospital, Chandigarh 160 030, India is not seen in up to one-third of neonates, thus necessitating the deployment of additional therapeutic options [2].

High pulmonary resistance is the main but not the only circulatory alteration in PPHN. Variable degrees of left ventricular dysfunction, systemic hypotension, and right ventricular dysfunction frequently co-exist and need to be detected to guide the therapeutic choices. Dopamine and adrenaline are nonselective inotropic agents and cause constriction of both systemic and pulmonary circulation. In addition, after prolonged hypoxia and cellular acidosis, systemic circulation may become unresponsive to catecholamines. Arginine vasopressin (AVP) has emerged as a promising agent in treatment of PPHN. AVP acts through V1 receptors on the systemic vascular smooth muscle cells causing non-catecholaminedependent vasoconstriction [4]. In addition, AVP is postulated to cause selective pulmonary vasodilatation through endothelium mediated NO induction. A number of case series have reported successful use of AVP in PPHN with catecholamineresistant hypotension. In this issue of the journal, Khare et al. present a case series of PPHN in which AVP was used for management [5]. Sixteen of the 18 neonates administered AVP responded with improvement in blood pressure, acidosis, and oxygenation. The evidence provided by the study is limited due to lack of a control group, retrospective design, and nonavailability of unique hemodynamic changes in the enrolled neonates. Yet, the study adds to accumulating evidence supporting the use of AVP in management of PPHN. Sildenafil, milrinone, vasopressin, and dobutamine make the armamentarium coming to rescue of clinicians and neonates with PPHN in resource-limited settings without access to iNO [6]. Apart from randomized controlled trials, which are needed to study efficacy of this approach, studies need to report the complete cohorts of PPHN with the profile of hemodynamic changes, response rate to different therapeutic agents, and risk factors of success or failure.

\section{Declarations}

Conflict of Interest None. 


\section{References}

1. Lai M-Y, Chu S-M, Lakshminrusimha S, Lin H-C. Beyond the inhaled nitric oxide in persistent pulmonary hypertension of the newborn. Pediatr Neonatol. 2018;59:15-23.

2. Jain A, McNamara PJ. Persistent pulmonary hypertension of the newborn: advances in diagnosis and treatment. Semin Fetal Neonatal Med. 2015;20:262-71.

3. Nakwan N, Jain S, Kumar K, et al. An Asian multicenter retrospective study on persistent pulmonary hypertension of the newborn: incidence, etiology, diagnosis, treatment and outcome. J Matern Fetal Neonatal Med. 2020;33:2032-7.
4. Tayama E, Ueda T, Shojima T, et al. Arginine vasopressin is an ideal drug after cardiac surgery for the management of low systemic vascular resistant hypotension concomitant with pulmonary hypertension. Interact Cardiovasc Thorac Surg. 2007;6:715-9.

5. Khare C, Adhisivam B, Bhat BV, Vaishnav D. Utility of low dose vasopressin for persistent pulmonary hypertension of newborn with catecholamine refractory shock. Indian J Pediatr. 2020 Oct 8. https:// doi.org/10.1007/s12098-020-03519-1. Accessed 21 Feb 2021.

6. Kelly LE, Ohlsson A, Shah PS. Sildenafil for pulmonary hypertension in neonates. Cochrane Database Syst Rev. 2017;8:CD005494.

Publisher's Note Springer Nature remains neutral with regard to jurisdictional claims in published maps and institutional affiliations. 